\title{
Evolution equation for quantum entanglement
}

\author{
THOMAS KONRAD ${ }^{1}$, FERNANDO DE MELO ${ }^{2,3}$, MARKUS TIERSCH²,3, CHRISTIAN KASZTELAN ${ }^{4}$, \\ ADRIANO ARAGÃO2,5 AND ANDREAS BUCHLEITNER ${ }^{2,3 *}$
}

\author{
${ }^{1}$ Quantum Research Group, School of Physics, University of KwaZulu-Natal, Private Bag X54001, Durban 4000, South Africa \\ ${ }^{2}$ Max-Planck-Institut für Physik komplexer Systeme, Nöthnitzer Str. 38, D-01187 Dresden, Germany \\ ${ }^{3}$ Albert-Ludwigs-Universität Freiburg, Physikalisches Institut, Hermann-Herder-Strasse 3, D-79104 Freiburg, Germany \\ ${ }^{4}$ Institut für Theoretische Physik C, RWTH Aachen, D-52056 Aachen, Germany \\ ${ }^{5}$ Instituto de Física, Universidade Federal do Rio de Janeiro, Caixa Postal 68.528, CEP 21945-970, Rio de Janeiro, RJ, Brazil \\ *e-mail: abu@pks.mpg.de
}

Quantum information technology ${ }^{1}$ largely relies on a precious and fragile resource, quantum entanglement, a highly nontrivial manifestation of the coherent superposition of states of composite quantum systems. However, our knowledge of the time evolution of this resource under realistic conditions-that is, when corrupted by environment-induced decoherence-is so far limited, and general statements on entanglement dynamics in open systems are scarce $^{2-11}$. Here we prove a simple and general factorization law for quantum systems shared by two parties, which describes the time evolution of entanglement on passage of either component through an arbitrary noisy channel. The robustness of entanglement-based quantum information processing protocols is thus easily and fully characterized by a single quantity.

Whenever we contemplate the potential technological applications of quantum information theory ${ }^{1}$, from secure quantum communication to quantum teleportation ${ }^{12}$, to quantum computation $^{13}$, we need to worry about the unavoidable and detrimental coupling of any such quantum device to uncontrolled degrees of freedom-typically lumped together under the label 'environment'. Coupling to the environment induces decoherence ${ }^{14-16}$; that is, it gradually destroys the phase relationship between quantum states, and thus their ability to interfere. In composite quantum systems, these phase relationships (or 'coherences') are at the origin of strong quantum correlations between measurements on distinct system constituents-which then are entangled. The promises of quantum information technology rely on exploring precisely these nonclassical correlations.

Yet, entanglement is not equivalent to many-particle coherences: it is an even stronger property, and hard to quantifyall commonly accepted entanglement measures ${ }^{17}$ are nonlinear functions of the density matrix, which describes the state of the composite quantum system, and in particular the coherences. Although an elaborate theory on the time evolution of quantum states under environment coupling is to hand, virtually no general results on entanglement dynamics have been stated. Hitherto, the time evolution of entanglement always needed to be deduced from the time evolution of the state $e^{2-10}$. In the present letter, a direct relationship between the initial and final entanglements of an arbitrary bipartite state of two qubits (the basic units of quantum information) subject to incoherent dynamics in one system component is derived, which, as illustrated in Fig. 1, renders the solution of the corresponding state evolution equation obsolete. Our result can be directly applied to input/output processes, such as gates used in sequential quantum computing. Moreover, it enables us to infer the evolution of entanglement under certain time-continuous influences of the environment, such as phase and amplitude damping.

Let us consider entangled states of qubit pairs, with one qubit being subject to an arbitrary channel $\$$-which may represent the influence of an environment, of a measurement or of both. To illustrate the situation, we consider a source that emits a particle to the left and another one to the right. Each particle on its own carries one qubit of quantum information (in general a superposition or mixture of two basis states $|0\rangle$ and $|1\rangle)$. We therefore also refer to the particles as 'left' and 'right' qubits. Let the particles leaving the source be in a pure state $|\chi\rangle$ :

$$
|\chi\rangle=\sqrt{\omega}|00\rangle+\sqrt{1-\omega}|11\rangle,
$$

with $0 \leq \omega \leq 1$; that is, for values of $\omega$ between zero and one the particles are in a coherent superposition of both being in state $|0\rangle$ and both being in state $|1\rangle$. Any pure state can be written in this form, modulo local unitary operations, and without loss of generality (see the Methods section).

We quantify entanglement by concurrence ${ }^{18} C$, which implies for the state $|\chi\rangle$ that $C(|\chi\rangle)=2 \sqrt{\omega(1-\omega)}$. For $\omega$ in (1) equal to zero or one, the state's entanglement and hence its concurrence vanishes; $\omega=1 / 2$ implies $|\chi\rangle=\left|\phi^{+}\right\rangle$, one of the maximally entangled Bell states, with maximal concurrence one.

Now the right qubit traverses an arbitrary quantum channel $\$$, as illustrated in Fig. 2a, and we want to derive the qubits' entanglement hereafter. To do so, note that the qubits' final state must be the same as in a dual picture ${ }^{19}$, where the roles of initial state and channel are interchanged, as depicted in Fig. 2b. Thus, the two-qubit state $|\chi\rangle$ is identified with a qubit channel $\$_{\chi}$, and the qubit channel \$ with a two-qubit state $\rho_{\$}$. Symbolically:

$$
\frac{(\mathbb{1} \otimes \$)|\chi\rangle\langle\chi|}{p^{\prime}}=\frac{\left(\$_{\chi} \otimes \mathbb{1}\right) \rho_{\$}}{p} .
$$




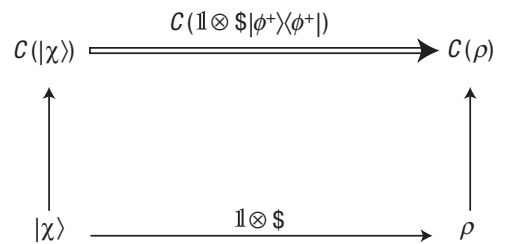

Figure 1 From state evolution to entanglement dynamics. Hitherto, the time evolution of entanglement $C$ under open system dynamics had to be deduced from the time evolution of the state $|\chi\rangle$. However, it turns out that knowledge of the entanglement evolution of the maximally entangled state $\left|\phi^{+}\right\rangle$under the channel $1 \otimes \$$ suffices to establish a direct mapping of $C(|\chi\rangle)$ onto $C(\rho)$, without detour via the state.

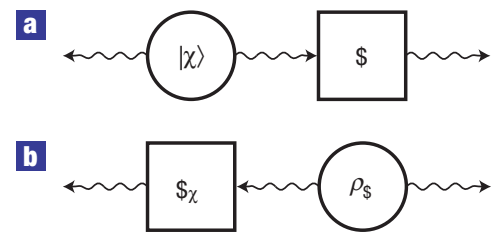

Figure 2 Entanglement decay scenarios. a, Laboratory scenario: one of the qubits of the initial state $|\chi\rangle$, the 'right' one, undergoes the action of a general quantum channel $\$$. b. Dual scenario: the same end result is obtained by interchanging the role of states and channels: the 'left' qubit of a mixed state $\rho_{\S}$ undergoes the action of the quantum channel $\$$. Circles represent sources of entangled states; squares symbolize channels.

Here, $p^{\prime}=\operatorname{Tr}[(\mathbb{1} \otimes \$)|\chi\rangle\langle\chi|]$ and $p=\operatorname{Tr}\left[\left(\$_{\chi} \otimes \mathbb{1}\right) \rho_{s}\right]$ are the probabilities for channels $\$$ and $\$_{\chi}$ to act on the states $|\chi\rangle\langle\chi|$ and $\rho_{\mathrm{s}}$, respectively. Thus, we also account for non-trace-preserving channels, where the particle number is not conserved.

We now need to determine $\$_{x}$ and $\rho_{\$}$ explicitly. For this purpose, we first remember that quantum teleportation ${ }^{12}$ is a means to transfer the state of one system to another one, in principle with perfect fidelity. Consequently, teleporting the right qubit of the state $|\chi\rangle$ assisted by the maximally entangled state $\left|\phi^{+}\right\rangle$leaves the state $|\chi\rangle$ invariant. This invariance is depicted in Fig. 3. We therefore obtain the same final state as in the situation we have considered so far (Fig. 2a) - if we replace the sourcepreparing state $|\chi\rangle$ by a source that prepares $|\chi\rangle$ followed by a teleportation of the right qubit as shown in Fig. 4. Let us now consider the source of the qubit pair in state $\left|\phi^{+}\right\rangle$, which we inserted with the teleportation. The succession of processes influencing the left qubit and those acting on the right qubit of the pair can be altered without consequences for the final state. For this reason we can replace the source producing the state $\left|\phi^{+}\right\rangle$together with the channel $\$$ acting on the right qubit by yet another source, which immediately prepares the state

$$
\rho_{\$}:=(\mathbb{1} \otimes \$)\left|\phi^{+}\right\rangle\left\langle\phi^{+}\right| / p^{\prime \prime},
$$

where $p^{\prime \prime}=\operatorname{Tr}\left[(\mathbb{1} \otimes \$)\left|\phi^{+}\right\rangle\left\langle\phi^{+}\right|\right]$; see Fig. 4 . The resulting scheme in Fig. 5 transfers entanglement between the qubit pairs prepared in states $|\chi\rangle$ and $\rho_{\mathrm{s}}$ to entanglement between the left qubit of the first pair and the right qubit of the second pair. This scheme is called entanglement swapping ${ }^{12,20,21}$.

Finally, we define $\$_{x}$ to be the channel corresponding to the change of the left qubit of $\rho_{\S}$ in Fig. 5, which includes a projection

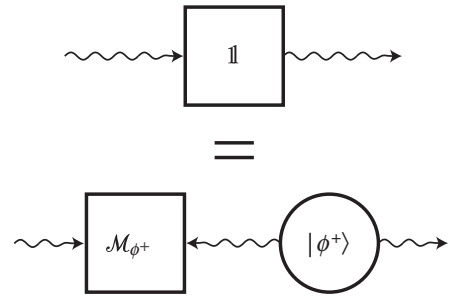

Figure 3 Quantum teleportation identity. The teleportation protocol transfers the incoming state from the left to the outgoing state on the right. The procedure consists of a composite Bell measurement on the incoming qubit and on one qubit of an auxiliary, maximally entangled state $\left|\phi^{+}\right\rangle$. We restrict ourselves to the case where the measurement results in a projection $\mathcal{M}_{\phi^{+}}$on the state $\left|\phi^{+}\right\rangle$. The remaining three possible measurement outcomes would imply local operations on the outgoing qubit, which can be incorporated in the action of the channel \$.

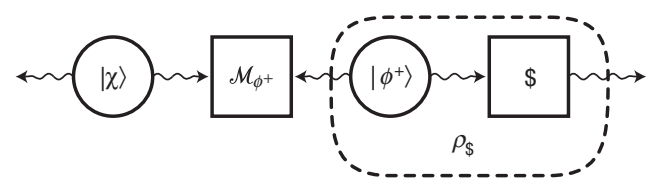

Figure 4 Building in the teleportation identity. The right qubit of $|\chi\rangle$ undergoes the action of a quantum channel $\$$, after intermediate teleportation. The maximally entangled state $\left|\phi^{+}\right\rangle$together with the action of the channel $1 \otimes \otimes$ yield the source of the mixed state $\rho_{\$}$.

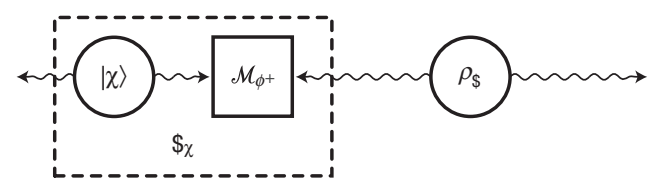

Figure 5 Entanglement swapping. Transfer of entanglement between the qubit pairs of $|\chi\rangle$ and $\rho_{\S}$, respectively, to entanglement between the outgoing pair of qubits. The Bell measurement $\mathcal{M}_{\phi^{+}}$together with the source of the entangled state $|\chi\rangle$ constitute the quantum channel $\$_{\chi}$ for the left qubit of state $\rho_{\S}$.

$\mathcal{M}_{\phi^{+}}$of the left qubit of state $\rho_{\S}$ and the right qubit of $|\chi\rangle$ on $\left|\phi^{+}\right\rangle$. Channel $\$_{\chi}$ can be interpreted as imperfect teleportation assisted by state $|\chi\rangle$, leaves the resulting state in general non-normalized and can be expressed in the particularly simple form

$$
\left(\$_{\chi} \otimes \mathbb{1}\right) \rho_{\S}=(M \otimes \mathbb{1}) \rho_{\$}\left(M^{\dagger} \otimes \mathbb{1}\right),
$$

with $M=(\sqrt{\omega}|0\rangle\langle 0|+\sqrt{1-\omega}| 1\rangle\langle 1|) / \sqrt{2}$. The normalized final state $\left(\$_{\chi} \otimes \mathbb{1}\right) \rho_{\$} / p$ is the same as $(\mathbb{1} \otimes \$)|\chi\rangle\langle\chi| / p^{\prime}$, as spelled out by (2) and in Fig. 2, but the entanglement evolution induced by the particular channel $\$_{x}$ can be deduced more easily, as we will now demonstrate. The concurrence $C$ of the final state $\rho^{\prime}=(\mathbb{1} \otimes \$)|\chi\rangle\langle\chi| / p^{\prime}$ is given by

$$
C\left(\rho^{\prime}\right)=\max \left\{0, \sqrt{\xi_{1}}-\sqrt{\xi_{2}}-\sqrt{\xi_{3}}-\sqrt{\xi_{4}}\right\},
$$

where the $\xi_{i}$ are the eigenvalues of the matrix $\rho^{\prime} \cdot \tilde{\rho}^{\prime}$, in decreasing order, with $\tilde{\rho}^{\prime}=\left(\sigma_{y} \otimes \sigma_{y}\right) \cdot \rho^{\prime *} \cdot\left(\sigma_{y} \otimes \sigma_{y}\right)$ and $\rho^{\prime *}$ the complex 
conjugate of $\rho^{\prime}$, in the canonical basis. In order to evaluate this expression, we use relation (2) together with (3). We write explicitly

$$
\rho^{\prime} \cdot \tilde{\rho}^{\prime}=\frac{1}{p^{2}}(M \otimes \mathbb{1}) \rho_{\$} \cdot\left[M \sigma_{y} M \otimes \sigma_{y}\right] \cdot \rho_{\$}^{*} \cdot\left[M \sigma_{y} \otimes \sigma_{y}\right],
$$

where we used that $M=M^{\dagger}=M^{*}$. For invertible $M$, it follows that the eigenvalues of $\rho^{\prime} \cdot \tilde{\rho}^{\prime}$ and $\rho_{\$} \cdot \tilde{\rho}_{\$}$ are proportional, because

$$
\begin{aligned}
& \operatorname{det}\left[\rho^{\prime} \cdot \tilde{\rho}^{\prime}-\xi \mathbb{1}\right] \\
= & \operatorname{det}\left[(M \otimes \mathbb{1})^{-1}\right] \operatorname{det}[M \otimes \mathbb{1}] \operatorname{det}\left[\rho^{\prime} \cdot \tilde{\rho}^{\prime}-\xi \mathbb{1}\right] \\
= & \operatorname{det}\left[(M \otimes \mathbb{1})^{-1} \rho^{\prime} \cdot \tilde{\rho}^{\prime}(M \otimes \mathbb{1})-\xi \mathbb{1}\right] \\
= & \operatorname{det}\left[\frac{1}{4 p^{2}} \omega(1-\omega) \rho_{\$} \cdot \tilde{\rho}_{\$}-\xi \mathbb{1}\right],
\end{aligned}
$$

where we used $M \sigma_{y} M=\sqrt{\omega(1-\omega)} \sigma_{y} / 2$ to obtain the last equality. Otherwise, for $\omega=0$ or $\omega=1$, concurrence vanishes, because $M \sigma_{y} M=0$.

Equation (4), together with the definitions of $\rho_{\$}=$ $(\mathbb{1} \otimes \$)\left|\phi^{+}\right\rangle\left\langle\phi^{+}\left|/ p^{\prime \prime}, \quad \rho^{\prime}=(\mathbb{1} \otimes \$)\right| \chi\right\rangle\langle\chi| / p^{\prime}$ and $C(|\chi\rangle)=$ $2 \sqrt{\omega(1-\omega)}$, thus leads to our central result:

$$
C[(\mathbb{1} \otimes \$)|\chi\rangle\langle\chi|]=C\left[(\mathbb{1} \otimes \$)\left|\phi^{+}\right\rangle\left\langle\phi^{+}\right|\right] C(|\chi\rangle) .
$$

The entanglement reduction under a one-sided noisy channel is independent of the initial state $|\chi\rangle$ and completely determined by the channel's action on the maximally entangled state. Thus, if we know the time evolution of the Bell state's entanglement, we know it for any pure initial state. This result can also be interpreted in terms of entanglement swapping between a pure state $|\chi\rangle$ and a mixed state $\rho_{\$}$, leading to the final state $\rho^{\prime}$, owing to the equivalence of the processes represented in Figs 2 and 5.

The factorization law (5) can be generalized for mixed initial states $\rho_{0}$, by virtue of the convexity of entanglement monotones such as concurrence, and given an optimal pure state decomposition $\rho_{0}=\sum_{j} p_{j}\left|\psi_{j}\right\rangle\left\langle\psi_{j}\right|$, in the sense that the average concurrence over this pure state decomposition is minimal ${ }^{22}$. It then immediately follows, by convexity, that $C\left[(\mathbb{1} \otimes \$) \rho_{0}\right]=C\left[\sum_{j} p_{j}(\mathbb{1} \otimes \$)\left|\psi_{j}\right\rangle\left\langle\psi_{j}\right|\right] \leq \sum_{j} p_{j} C\left[(\mathbb{1} \otimes \$)\left|\psi_{j}\right\rangle\left\langle\psi_{j}\right|\right]$, and application of (5) leaves us with

$$
C\left[(\mathbb{1} \otimes \$) \rho_{0}\right] \leq C\left[(\mathbb{1} \otimes \$)\left|\phi^{+}\right\rangle\left\langle\phi^{+}\right|\right] C\left(\rho_{0}\right) .
$$

This inequality holds for all one-sided channels \$, and has an immediate generalization for local two-sided channels $\$_{1} \otimes \$_{2}=\left(\$_{1} \otimes \mathbb{1}\right)\left(\mathbb{1} \otimes \$_{2}\right):$

$$
\begin{aligned}
C\left[\left(\$_{1} \otimes \$_{2}\right) \rho_{0}\right] \leq & C\left[\left(\$_{1} \otimes \mathbb{1}\right)\left|\phi^{+}\right\rangle\left\langle\phi^{+}\right|\right] \\
& \times C\left[\left(\mathbb{1} \otimes \$_{2}\right)\left|\phi^{+}\right\rangle\left\langle\phi^{+}\right|\right] C\left(\rho_{0}\right) .
\end{aligned}
$$

The concurrence after passage through a two-sided channel is thus bounded from above, which immediately implies a sufficient criterion for finite-time disentanglement ${ }^{2,3,6}$ of arbitrary initial states, in terms of the evolution of the concurrence of the maximally entangled state under either one of the one-sided channels (for example, choose $\$_{1}$ or $\$_{2}$ induced by infinite temperature or depolarizing environments).

Let us finally identify relevant cases when the equality in (6) holds. For this purpose, we consider mixed states that are obtained after the application of a one-sided channel to an arbitrary pure state, $\rho_{0}=(\mathbb{1} \otimes \$)\left|\psi_{0}\right\rangle\left\langle\psi_{0}\right|$. This occurs, for instance, if the qubit originally prepared in a pure state suffers amplitude decay, and the resulting mixed state again is subject to decay dynamics. This is tantamount to the concatenation of channels on one side, $\left(\mathbb{1} \otimes \$_{2}\right)\left(\mathbb{1} \otimes \$_{1}\right)\left|\psi_{0}\right\rangle\left\langle\psi_{0}\right|$, which can be lumped together as one channel that combines both actions, $\left(\mathbb{1} \otimes \$_{2}\right)\left(\mathbb{1} \otimes \$_{1}\right)=\mathbb{1} \otimes \$_{2,1}$. In a similar vein as for (7), and using the factorization relation (5) for pure states, we deduce

$$
\begin{gathered}
C\left[\left(\mathbb{1} \otimes \$_{2,1}\right)\left|\phi^{+}\right\rangle\left\langle\phi^{+}\right|\right] C\left(\left|\psi_{0}\right\rangle\right) \leq C\left[\left(\mathbb{1} \otimes \$_{2}\right)\left|\phi^{+}\right\rangle\left\langle\phi^{+}\right|\right] \\
\times C\left[\left(\mathbb{1} \otimes \$_{1}\right)\left|\phi^{+}\right\rangle\left\langle\phi^{+}\right|\right] C\left(\left|\psi_{0}\right\rangle\right) .
\end{gathered}
$$

The initial state's concurrence rescales both sides of the equation by the same amount, and therefore does not matter. It is now sufficient to investigate the time dependence of the maximally entangled state's concurrence under the concatenated channels (much as for the evaluation of (5)): if all of these are of the form $C(t)=\exp (-\Gamma t)$ (which is the case, for example, for $\$_{1}$ being an amplitude decay and $\$_{2}$ a dephasing channel), then the equality holds in (6), with $\$=\$_{1}, \rho_{0}=\left(\mathbb{1} \otimes \$_{2}\right)\left|\psi_{0}\right\rangle\left\langle\psi_{0}\right|$ and $C\left(\rho_{t}\right)=\exp \left(-\Gamma_{1} t\right) C\left(\rho_{0}\right)$ (and, equivalently, for the roles of channels 1 and 2 interchanged).

In conclusion, equations (5)-(8) provide us with the first closed expression for the time evolution of an entangled qubit pair under general local, single- and two-sided channels, without recourse to the time evolution of the underlying quantum state itself. This is a general result inherited from the Jamiołkowski isomorphism ${ }^{19}$, which is here 'lifted' from state to entanglement evolution (see Fig. 1), and eases the experimental characterization of entanglement dynamics under unknown channels dramatically: instead of exploring the time-dependent action of the channel on all initial states, it suffices to probe the entanglement evolution of the maximally entangled state alone.

Because the Jamiołkowski isomorphism extends to bipartite systems of arbitrary finite dimension, the dual picture used here for qubit pairs will prevail in higher dimensions. The maximally entangled state will therefore retain its role in the evolution equation for entanglement dynamics. However, because a closed algebraic expression like (4) is hitherto unavailable for mixed-state entanglement in higher dimensions ${ }^{17}$, the derivation of a higherdimensional analogue of (5) will have to follow a different strategy.

\section{METHODS}

In our derivation of (5), we used $C(\alpha \rho)=\alpha C(\rho)$, which follows right from the definition of pure state concurrence ${ }^{17,18}$, and $p^{\prime}=4 p p^{\prime \prime}$. The latter can be derived by careful comparison of the individual normalization factors. In order to compute the entanglement of the normalized final state, $C[(\mathbb{1} \otimes \$)|\chi\rangle\langle\chi|]$ has to be divided by $p^{\prime}$.

Furthermore, note that our choice (1) of $|\chi\rangle$ in the Schmidt representation does not restrict the generality of relation (5). Using a general pure state $\left|\chi^{\prime}\right\rangle\left\langle\chi^{\prime}\left|=\left(\mathcal{U}_{a} \otimes \mathcal{U}_{b}\right)\right| \chi\right\rangle\langle\chi|$ as the initial state leads to the same result (5), because unitary operation $\mathcal{U}_{a}$ does not affect the concurrence of the final state, and unitary operation $\mathcal{U}_{b}$ can be incorporated into the channel: $C\left[(\mathbb{1} \otimes \$)\left|\chi^{\prime}\right\rangle\left\langle\chi^{\prime}\right|\right]=C\left[\left(\mathbb{1} \otimes \$ \mathcal{U}_{b}\right)|\chi\rangle\langle\chi|\right]=C\left[\left(\mathbb{1} \otimes \$ \mathcal{U}_{b}\right)\left|\phi^{+}\right\rangle\left\langle\phi^{+}\right|\right] C(|\chi\rangle)=$ $C\left[(\mathbb{1} \otimes \$)\left|\phi^{+}\right\rangle\left\langle\phi^{+}\right|\right] C\left(\left|\chi^{\prime}\right\rangle\right)$.

Received 17 August 2007; accepted 22 November 2007; published 23 December 2007.

\section{References}

1. Bennett, C. H. \& DiVincenzo, D. P. Quantum information and computation. Nature 404, $247-255$ (2000).

2. Życzkowski, K., Horodecki, P., Horodecki, M. \& Horodecki, R. Dynamics of quantum entanglement. Phys. Rev. A 65, 012101 (2001).

3. Dodd, P. J. \& Halliwell, J. J. Disentanglement and decoherence by open system dynamics. Phys. Rev. A 69, 052105 (2004)

4. Roos, C. F. et al. Bell states of atoms with ultralong lifetimes and their tomographic state analysis. Phys. Rev. Lett. 92, 220402 (2004).

5. Carvalho, A. R. R., Mintert, F. \& Buchleitner, A. Decoherence and multipartite entanglement. Phys. Rev. Lett. 93, 230501 (2004).

6. Yu, T. \& Eberly, J. H. Quantum open system theory: Bipartite aspects. Phys. Rev. Lett. 97, 140403 (2006). 
7. Santos, M. F., Milman, P., Davidovich, L. \& Zagury, N. Direct measurement of finite-time disentanglement induced by a reservoir. Phys. Rev. A 73, 040305 (2006).

8. Dür, W. \& Briegel, H.-J. Stability of macroscopic entanglement under decoherence. Phys. Rev. Lett. 92, 180403 (2004).

9. Almeida, M. P. et al. Environment-induced sudden death of entanglement. Science 316, 579-582 (2007).

10. Carvalho, A. R. R., Busse, M., Brodier, O., Viviescas, C. \& Buchleitner, A. Optimal dynamical characterization of entanglement. Phys. Rev. Lett. 98, 190501 (2007).

11. Horodecki, M., Shor, P. W. \& Ruskai, M. B. General entanglement breaking channels. Rev. Math Phys. 15, 629-641 (2003).

12. Bennett, C. H. et al. Teleporting an unknown quantum state via dual classical and Einstein-Podolsky-Rosen channels. Phys. Rev. Lett. 70, 1895-1899 (1993).

13. Nielsen, M. A. \& Chuang, I. L. Quantum Computation and Quantum Information (Cambridge Univ. Press, Cambridge, 2000).

14. Schlosshauer, M. Decoherence, the measurement problem, and interpretations of quantum mechanics. Rev. Mod. Phys. 76, 1267-1306 (2004).

15. Zurek, W. H. Decoherence, einselection, and the quantum origins of the classical. Rev. Mod. Phys. 75, 715-776 (2003).

16. Joos, E. et al. Decoherence and the Appearance of a Classical World in Quantum Theory (Springer, Berlin, 2003).
17. Mintert, F., Carvalho, A. R. R., Kuś, M. \& Buchleitner, A. Measures and dynamics of entangled states. Phys. Rep. 415, 207-259 (2005).

18. Wootters, W. K. Entanglement of formation of an arbitrary state of two qubits. Phys. Rev. Lett. 80, 2245-2248 (1998).

19. Jamiołkowski, A. Linear transformations which preserve trace and positive semidefiniteness of operators. Rep. Math. Phys. 3, 275-278 (1972).

20. Żukowski, M., Zeilinger, A., Horne, M. A. \& Ekert, A. K. "Event-ready-detectors" Bell experiment via entanglement swapping. Phys. Rev. Lett. 71, 4287-4290 (1993).

21. Pan, J.-W., Bouwmeester, D., Weinfurter, H. \& Zeilinger, A. Experimental entanglement swapping: Entangling photons that never interacted. Phys. Rev. Lett. 80, 3891-3894 (1998).

22. Bennett, C. H., DiVincenzo, D. P., Smolin, J. A. \& Wootters, W. K. Mixed-state entanglement and quantum error correction. Phys. Rev. A 54, 3824-3851 (1996).

\section{Acknowledgements}

We thank J. Audretsch, H.-P. Breuer, L. Davidovich, L. Diosi and C. Viviescas for discussions. Partial support by DAAD/CAPES under a PROBRAL grant, as well as VolkswagenStiftung, is gratefully acknowledged.

Correspondence and requests for materials should be addressed to A.B.

Reprints and permission information is available online at http://npg.nature.com/reprintsandpermissions/ 